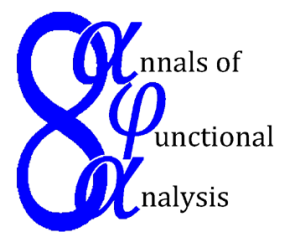

Ann. Funct. Anal. 7 (2016), no. 1, 136-149

http://dx.doi.org/10.1215/20088752-3428247

ISSN: 2008-8752 (electronic)

http://projecteuclid.org/afa

\title{
COMMUTING CONTRACTIVE IDEMPOTENTS IN MEASURE ALGEBRAS
}

\author{
NICO SPRONK \\ Dedicated to Professor Anthony To-Ming Lau, in honor of his contributions to, and leadership \\ in, the international abstract harmonic analysis community, the Canadian mathematical \\ community, and my career
}

Communicated by K. F. Taylor

\begin{abstract}
We determine when contractive idempotents in the measure algebra of a locally compact group commute. We consider a dynamical version of the same result. We also look at some properties of groups of measures whose identity is a contractive idempotent.
\end{abstract}

Let $G$ be a locally compact group. When $G$ is abelian, Cohen [1] characterized all of the idempotents in the measure algebra $\mathrm{M}(G)$. For nonabelian $G$, the idempotent probabilities were characterized by Kawada and Itô [3], while the contractive idempotents were characterized by Greenleaf [2]. We give an exact statement of their results in Theorem 0.1 below. For certain compact groups, the central idempotent measures were characterized by Rider [7], in a manner which is pleasingly reminiscent of Cohen's result on abelian groups. Rider points out a counterexample to his result when some assumptions are dropped. This has motivated our Example 1.3(i) below.

Discussion of contactive idempotents has been conducted in the setting of locally compact quantum groups by Neufang, Salmi, Skalski, and the present author [5].

Copyright 2016 by the Tusi Mathematical Research Group.

Received Mar. 15, 2015; Accepted Aug. 11, 2015.

2010 Mathematics Subject Classification. Primary 43A05; Secondary 43A77, 43A40.

Keywords. measure algebra, idempotent, groups of measures. 


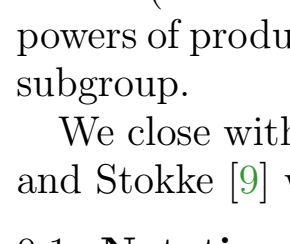

Ann. Funct. Anal. 7 (2016), no. 1, 136-149

http://dx.doi.org/10.1215/20088752-3428247

ISSN: 2008-8752 (electronic)

http://projecteuclid.org/afa

\title{
COMMUTING CONTRACTIVE IDEMPOTENTS IN MEASURE ALGEBRAS
}

\author{
NICO SPRONK \\ Dedicated to Professor Anthony To-Ming Lau, in honor of his contributions to, and leadership \\ in, the international abstract harmonic analysis community, the Canadian mathematical \\ community, and my career
}

Communicated by K. F. Taylor

\begin{abstract}
We determine when contractive idempotents in the measure algebra of a locally compact group commute. We consider a dynamical version of the same result. We also look at some properties of groups of measures whose identity is a contractive idempotent.
\end{abstract}

Let $G$ be a locally compact group. When $G$ is abelian, Cohen [1] characterized all of the idempotents in the measure algebra $\mathrm{M}(G)$. For nonabelian $G$, the idempotent probabilities were characterized by Kawada and Itô [3], while the contractive idempotents were characterized by Greenleaf [2]. We give an exact statement of their results in Theorem 0.1 below. For certain compact groups, the central idempotent measures were characterized by Rider [7], in a manner which is pleasingly reminiscent of Cohen's result on abelian groups. Rider points out a counterexample to his result when some assumptions are dropped. This has motivated our Example 1.3(i) below.

Discussion of contactive idempotents has been conducted in the setting of locally compact quantum groups by Neufang, Salmi, Skalski, and the present author [5].

Copyright 2016 by the Tusi Mathematical Research Group.

Received Mar. 15, 2015; Accepted Aug. 11, 2015.

2010 Mathematics Subject Classification. Primary 43A05; Secondary 43A77, 43A40.

Keywords. measure algebra, idempotent, groups of measures. 


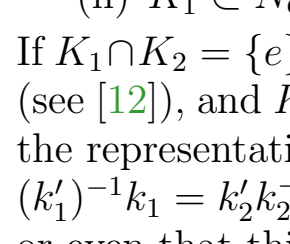

Ann. Funct. Anal. 7 (2016), no. 1, 136-149

http://dx.doi.org/10.1215/20088752-3428247

ISSN: 2008-8752 (electronic)

http://projecteuclid.org/afa

\title{
COMMUTING CONTRACTIVE IDEMPOTENTS IN MEASURE ALGEBRAS
}

\author{
NICO SPRONK \\ Dedicated to Professor Anthony To-Ming Lau, in honor of his contributions to, and leadership \\ in, the international abstract harmonic analysis community, the Canadian mathematical \\ community, and my career
}

Communicated by K. F. Taylor

\begin{abstract}
We determine when contractive idempotents in the measure algebra of a locally compact group commute. We consider a dynamical version of the same result. We also look at some properties of groups of measures whose identity is a contractive idempotent.
\end{abstract}

Let $G$ be a locally compact group. When $G$ is abelian, Cohen [1] characterized all of the idempotents in the measure algebra $\mathrm{M}(G)$. For nonabelian $G$, the idempotent probabilities were characterized by Kawada and Itô [3], while the contractive idempotents were characterized by Greenleaf [2]. We give an exact statement of their results in Theorem 0.1 below. For certain compact groups, the central idempotent measures were characterized by Rider [7], in a manner which is pleasingly reminiscent of Cohen's result on abelian groups. Rider points out a counterexample to his result when some assumptions are dropped. This has motivated our Example 1.3(i) below.

Discussion of contactive idempotents has been conducted in the setting of locally compact quantum groups by Neufang, Salmi, Skalski, and the present author [5].

Copyright 2016 by the Tusi Mathematical Research Group.

Received Mar. 15, 2015; Accepted Aug. 11, 2015.

2010 Mathematics Subject Classification. Primary 43A05; Secondary 43A77, 43A40.

Keywords. measure algebra, idempotent, groups of measures. 


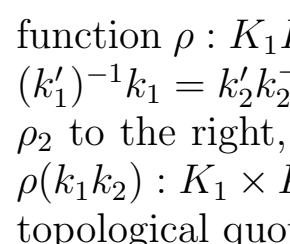

Ann. Funct. Anal. 7 (2016), no. 1, 136-149

http://dx.doi.org/10.1215/20088752-3428247

ISSN: 2008-8752 (electronic)

http://projecteuclid.org/afa

\title{
COMMUTING CONTRACTIVE IDEMPOTENTS IN MEASURE ALGEBRAS
}

\author{
NICO SPRONK \\ Dedicated to Professor Anthony To-Ming Lau, in honor of his contributions to, and leadership \\ in, the international abstract harmonic analysis community, the Canadian mathematical \\ community, and my career
}

Communicated by K. F. Taylor

\begin{abstract}
We determine when contractive idempotents in the measure algebra of a locally compact group commute. We consider a dynamical version of the same result. We also look at some properties of groups of measures whose identity is a contractive idempotent.
\end{abstract}

Let $G$ be a locally compact group. When $G$ is abelian, Cohen [1] characterized all of the idempotents in the measure algebra $\mathrm{M}(G)$. For nonabelian $G$, the idempotent probabilities were characterized by Kawada and Itô [3], while the contractive idempotents were characterized by Greenleaf [2]. We give an exact statement of their results in Theorem 0.1 below. For certain compact groups, the central idempotent measures were characterized by Rider [7], in a manner which is pleasingly reminiscent of Cohen's result on abelian groups. Rider points out a counterexample to his result when some assumptions are dropped. This has motivated our Example 1.3(i) below.

Discussion of contactive idempotents has been conducted in the setting of locally compact quantum groups by Neufang, Salmi, Skalski, and the present author [5].

Copyright 2016 by the Tusi Mathematical Research Group.

Received Mar. 15, 2015; Accepted Aug. 11, 2015.

2010 Mathematics Subject Classification. Primary 43A05; Secondary 43A77, 43A40.

Keywords. measure algebra, idempotent, groups of measures. 


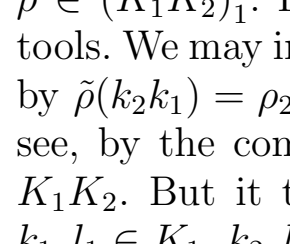

Ann. Funct. Anal. 7 (2016), no. 1, 136-149

http://dx.doi.org/10.1215/20088752-3428247

ISSN: 2008-8752 (electronic)

http://projecteuclid.org/afa

\title{
COMMUTING CONTRACTIVE IDEMPOTENTS IN MEASURE ALGEBRAS
}

\author{
NICO SPRONK \\ Dedicated to Professor Anthony To-Ming Lau, in honor of his contributions to, and leadership \\ in, the international abstract harmonic analysis community, the Canadian mathematical \\ community, and my career
}

Communicated by K. F. Taylor

\begin{abstract}
We determine when contractive idempotents in the measure algebra of a locally compact group commute. We consider a dynamical version of the same result. We also look at some properties of groups of measures whose identity is a contractive idempotent.
\end{abstract}

Let $G$ be a locally compact group. When $G$ is abelian, Cohen [1] characterized all of the idempotents in the measure algebra $\mathrm{M}(G)$. For nonabelian $G$, the idempotent probabilities were characterized by Kawada and Itô [3], while the contractive idempotents were characterized by Greenleaf [2]. We give an exact statement of their results in Theorem 0.1 below. For certain compact groups, the central idempotent measures were characterized by Rider [7], in a manner which is pleasingly reminiscent of Cohen's result on abelian groups. Rider points out a counterexample to his result when some assumptions are dropped. This has motivated our Example 1.3(i) below.

Discussion of contactive idempotents has been conducted in the setting of locally compact quantum groups by Neufang, Salmi, Skalski, and the present author [5].

Copyright 2016 by the Tusi Mathematical Research Group.

Received Mar. 15, 2015; Accepted Aug. 11, 2015.

2010 Mathematics Subject Classification. Primary 43A05; Secondary 43A77, 43A40.

Keywords. measure algebra, idempotent, groups of measures. 


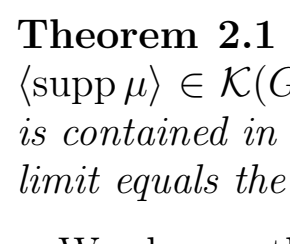

Ann. Funct. Anal. 7 (2016), no. 1, 136-149

http://dx.doi.org/10.1215/20088752-3428247

ISSN: 2008-8752 (electronic)

http://projecteuclid.org/afa

\title{
COMMUTING CONTRACTIVE IDEMPOTENTS IN MEASURE ALGEBRAS
}

\author{
NICO SPRONK \\ Dedicated to Professor Anthony To-Ming Lau, in honor of his contributions to, and leadership \\ in, the international abstract harmonic analysis community, the Canadian mathematical \\ community, and my career
}

Communicated by K. F. Taylor

\begin{abstract}
We determine when contractive idempotents in the measure algebra of a locally compact group commute. We consider a dynamical version of the same result. We also look at some properties of groups of measures whose identity is a contractive idempotent.
\end{abstract}

Let $G$ be a locally compact group. When $G$ is abelian, Cohen [1] characterized all of the idempotents in the measure algebra $\mathrm{M}(G)$. For nonabelian $G$, the idempotent probabilities were characterized by Kawada and Itô [3], while the contractive idempotents were characterized by Greenleaf [2]. We give an exact statement of their results in Theorem 0.1 below. For certain compact groups, the central idempotent measures were characterized by Rider [7], in a manner which is pleasingly reminiscent of Cohen's result on abelian groups. Rider points out a counterexample to his result when some assumptions are dropped. This has motivated our Example 1.3(i) below.

Discussion of contactive idempotents has been conducted in the setting of locally compact quantum groups by Neufang, Salmi, Skalski, and the present author [5].

Copyright 2016 by the Tusi Mathematical Research Group.

Received Mar. 15, 2015; Accepted Aug. 11, 2015.

2010 Mathematics Subject Classification. Primary 43A05; Secondary 43A77, 43A40.

Keywords. measure algebra, idempotent, groups of measures. 


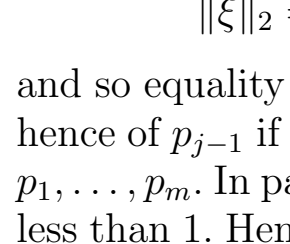

Ann. Funct. Anal. 7 (2016), no. 1, 136-149

http://dx.doi.org/10.1215/20088752-3428247

ISSN: 2008-8752 (electronic)

http://projecteuclid.org/afa

\title{
COMMUTING CONTRACTIVE IDEMPOTENTS IN MEASURE ALGEBRAS
}

\author{
NICO SPRONK \\ Dedicated to Professor Anthony To-Ming Lau, in honor of his contributions to, and leadership \\ in, the international abstract harmonic analysis community, the Canadian mathematical \\ community, and my career
}

Communicated by K. F. Taylor

\begin{abstract}
We determine when contractive idempotents in the measure algebra of a locally compact group commute. We consider a dynamical version of the same result. We also look at some properties of groups of measures whose identity is a contractive idempotent.
\end{abstract}

Let $G$ be a locally compact group. When $G$ is abelian, Cohen [1] characterized all of the idempotents in the measure algebra $\mathrm{M}(G)$. For nonabelian $G$, the idempotent probabilities were characterized by Kawada and Itô [3], while the contractive idempotents were characterized by Greenleaf [2]. We give an exact statement of their results in Theorem 0.1 below. For certain compact groups, the central idempotent measures were characterized by Rider [7], in a manner which is pleasingly reminiscent of Cohen's result on abelian groups. Rider points out a counterexample to his result when some assumptions are dropped. This has motivated our Example 1.3(i) below.

Discussion of contactive idempotents has been conducted in the setting of locally compact quantum groups by Neufang, Salmi, Skalski, and the present author [5].

Copyright 2016 by the Tusi Mathematical Research Group.

Received Mar. 15, 2015; Accepted Aug. 11, 2015.

2010 Mathematics Subject Classification. Primary 43A05; Secondary 43A77, 43A40.

Keywords. measure algebra, idempotent, groups of measures. 


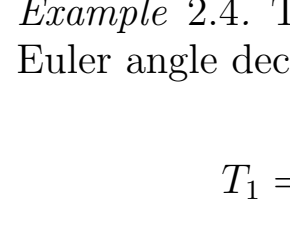

Ann. Funct. Anal. 7 (2016), no. 1, 136-149

http://dx.doi.org/10.1215/20088752-3428247

ISSN: 2008-8752 (electronic)

http://projecteuclid.org/afa

\title{
COMMUTING CONTRACTIVE IDEMPOTENTS IN MEASURE ALGEBRAS
}

\author{
NICO SPRONK \\ Dedicated to Professor Anthony To-Ming Lau, in honor of his contributions to, and leadership \\ in, the international abstract harmonic analysis community, the Canadian mathematical \\ community, and my career
}

Communicated by K. F. Taylor

\begin{abstract}
We determine when contractive idempotents in the measure algebra of a locally compact group commute. We consider a dynamical version of the same result. We also look at some properties of groups of measures whose identity is a contractive idempotent.
\end{abstract}

Let $G$ be a locally compact group. When $G$ is abelian, Cohen [1] characterized all of the idempotents in the measure algebra $\mathrm{M}(G)$. For nonabelian $G$, the idempotent probabilities were characterized by Kawada and Itô [3], while the contractive idempotents were characterized by Greenleaf [2]. We give an exact statement of their results in Theorem 0.1 below. For certain compact groups, the central idempotent measures were characterized by Rider [7], in a manner which is pleasingly reminiscent of Cohen's result on abelian groups. Rider points out a counterexample to his result when some assumptions are dropped. This has motivated our Example 1.3(i) below.

Discussion of contactive idempotents has been conducted in the setting of locally compact quantum groups by Neufang, Salmi, Skalski, and the present author [5].

Copyright 2016 by the Tusi Mathematical Research Group.

Received Mar. 15, 2015; Accepted Aug. 11, 2015.

2010 Mathematics Subject Classification. Primary 43A05; Secondary 43A77, 43A40.

Keywords. measure algebra, idempotent, groups of measures. 


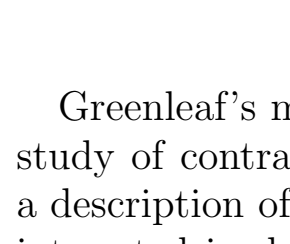

Ann. Funct. Anal. 7 (2016), no. 1, 136-149

http://dx.doi.org/10.1215/20088752-3428247

ISSN: 2008-8752 (electronic)

http://projecteuclid.org/afa

\title{
COMMUTING CONTRACTIVE IDEMPOTENTS IN MEASURE ALGEBRAS
}

\author{
NICO SPRONK \\ Dedicated to Professor Anthony To-Ming Lau, in honor of his contributions to, and leadership \\ in, the international abstract harmonic analysis community, the Canadian mathematical \\ community, and my career
}

Communicated by K. F. Taylor

\begin{abstract}
We determine when contractive idempotents in the measure algebra of a locally compact group commute. We consider a dynamical version of the same result. We also look at some properties of groups of measures whose identity is a contractive idempotent.
\end{abstract}

Let $G$ be a locally compact group. When $G$ is abelian, Cohen [1] characterized all of the idempotents in the measure algebra $\mathrm{M}(G)$. For nonabelian $G$, the idempotent probabilities were characterized by Kawada and Itô [3], while the contractive idempotents were characterized by Greenleaf [2]. We give an exact statement of their results in Theorem 0.1 below. For certain compact groups, the central idempotent measures were characterized by Rider [7], in a manner which is pleasingly reminiscent of Cohen's result on abelian groups. Rider points out a counterexample to his result when some assumptions are dropped. This has motivated our Example 1.3(i) below.

Discussion of contactive idempotents has been conducted in the setting of locally compact quantum groups by Neufang, Salmi, Skalski, and the present author [5].

Copyright 2016 by the Tusi Mathematical Research Group.

Received Mar. 15, 2015; Accepted Aug. 11, 2015.

2010 Mathematics Subject Classification. Primary 43A05; Secondary 43A77, 43A40.

Keywords. measure algebra, idempotent, groups of measures. 


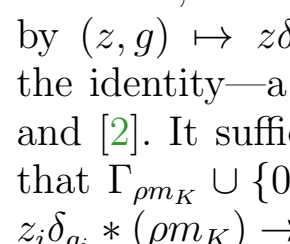

Ann. Funct. Anal. 7 (2016), no. 1, 136-149

http://dx.doi.org/10.1215/20088752-3428247

ISSN: 2008-8752 (electronic)

http://projecteuclid.org/afa

\title{
COMMUTING CONTRACTIVE IDEMPOTENTS IN MEASURE ALGEBRAS
}

\author{
NICO SPRONK \\ Dedicated to Professor Anthony To-Ming Lau, in honor of his contributions to, and leadership \\ in, the international abstract harmonic analysis community, the Canadian mathematical \\ community, and my career
}

Communicated by K. F. Taylor

\begin{abstract}
We determine when contractive idempotents in the measure algebra of a locally compact group commute. We consider a dynamical version of the same result. We also look at some properties of groups of measures whose identity is a contractive idempotent.
\end{abstract}

Let $G$ be a locally compact group. When $G$ is abelian, Cohen [1] characterized all of the idempotents in the measure algebra $\mathrm{M}(G)$. For nonabelian $G$, the idempotent probabilities were characterized by Kawada and Itô [3], while the contractive idempotents were characterized by Greenleaf [2]. We give an exact statement of their results in Theorem 0.1 below. For certain compact groups, the central idempotent measures were characterized by Rider [7], in a manner which is pleasingly reminiscent of Cohen's result on abelian groups. Rider points out a counterexample to his result when some assumptions are dropped. This has motivated our Example 1.3(i) below.

Discussion of contactive idempotents has been conducted in the setting of locally compact quantum groups by Neufang, Salmi, Skalski, and the present author [5].

Copyright 2016 by the Tusi Mathematical Research Group.

Received Mar. 15, 2015; Accepted Aug. 11, 2015.

2010 Mathematics Subject Classification. Primary 43A05; Secondary 43A77, 43A40.

Keywords. measure algebra, idempotent, groups of measures. 


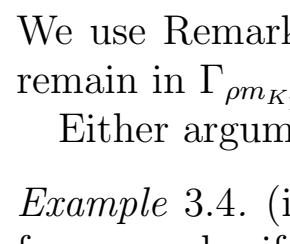

Ann. Funct. Anal. 7 (2016), no. 1, 136-149

http://dx.doi.org/10.1215/20088752-3428247

ISSN: 2008-8752 (electronic)

http://projecteuclid.org/afa

\title{
COMMUTING CONTRACTIVE IDEMPOTENTS IN MEASURE ALGEBRAS
}

\author{
NICO SPRONK \\ Dedicated to Professor Anthony To-Ming Lau, in honor of his contributions to, and leadership \\ in, the international abstract harmonic analysis community, the Canadian mathematical \\ community, and my career
}

Communicated by K. F. Taylor

\begin{abstract}
We determine when contractive idempotents in the measure algebra of a locally compact group commute. We consider a dynamical version of the same result. We also look at some properties of groups of measures whose identity is a contractive idempotent.
\end{abstract}

Let $G$ be a locally compact group. When $G$ is abelian, Cohen [1] characterized all of the idempotents in the measure algebra $\mathrm{M}(G)$. For nonabelian $G$, the idempotent probabilities were characterized by Kawada and Itô [3], while the contractive idempotents were characterized by Greenleaf [2]. We give an exact statement of their results in Theorem 0.1 below. For certain compact groups, the central idempotent measures were characterized by Rider [7], in a manner which is pleasingly reminiscent of Cohen's result on abelian groups. Rider points out a counterexample to his result when some assumptions are dropped. This has motivated our Example 1.3(i) below.

Discussion of contactive idempotents has been conducted in the setting of locally compact quantum groups by Neufang, Salmi, Skalski, and the present author [5].

Copyright 2016 by the Tusi Mathematical Research Group.

Received Mar. 15, 2015; Accepted Aug. 11, 2015.

2010 Mathematics Subject Classification. Primary 43A05; Secondary 43A77, 43A40.

Keywords. measure algebra, idempotent, groups of measures. 


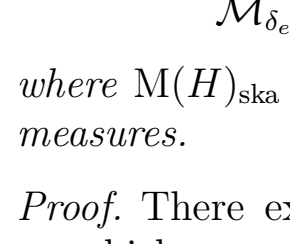

Ann. Funct. Anal. 7 (2016), no. 1, 136-149

http://dx.doi.org/10.1215/20088752-3428247

ISSN: 2008-8752 (electronic)

http://projecteuclid.org/afa

\title{
COMMUTING CONTRACTIVE IDEMPOTENTS IN MEASURE ALGEBRAS
}

\author{
NICO SPRONK \\ Dedicated to Professor Anthony To-Ming Lau, in honor of his contributions to, and leadership \\ in, the international abstract harmonic analysis community, the Canadian mathematical \\ community, and my career
}

Communicated by K. F. Taylor

\begin{abstract}
We determine when contractive idempotents in the measure algebra of a locally compact group commute. We consider a dynamical version of the same result. We also look at some properties of groups of measures whose identity is a contractive idempotent.
\end{abstract}

Let $G$ be a locally compact group. When $G$ is abelian, Cohen [1] characterized all of the idempotents in the measure algebra $\mathrm{M}(G)$. For nonabelian $G$, the idempotent probabilities were characterized by Kawada and Itô [3], while the contractive idempotents were characterized by Greenleaf [2]. We give an exact statement of their results in Theorem 0.1 below. For certain compact groups, the central idempotent measures were characterized by Rider [7], in a manner which is pleasingly reminiscent of Cohen's result on abelian groups. Rider points out a counterexample to his result when some assumptions are dropped. This has motivated our Example 1.3(i) below.

Discussion of contactive idempotents has been conducted in the setting of locally compact quantum groups by Neufang, Salmi, Skalski, and the present author [5].

Copyright 2016 by the Tusi Mathematical Research Group.

Received Mar. 15, 2015; Accepted Aug. 11, 2015.

2010 Mathematics Subject Classification. Primary 43A05; Secondary 43A77, 43A40.

Keywords. measure algebra, idempotent, groups of measures. 


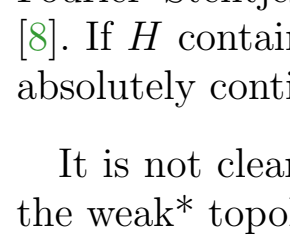

Ann. Funct. Anal. 7 (2016), no. 1, 136-149

http://dx.doi.org/10.1215/20088752-3428247

ISSN: 2008-8752 (electronic)

http://projecteuclid.org/afa

\title{
COMMUTING CONTRACTIVE IDEMPOTENTS IN MEASURE ALGEBRAS
}

\author{
NICO SPRONK \\ Dedicated to Professor Anthony To-Ming Lau, in honor of his contributions to, and leadership \\ in, the international abstract harmonic analysis community, the Canadian mathematical \\ community, and my career
}

Communicated by K. F. Taylor

\begin{abstract}
We determine when contractive idempotents in the measure algebra of a locally compact group commute. We consider a dynamical version of the same result. We also look at some properties of groups of measures whose identity is a contractive idempotent.
\end{abstract}

Let $G$ be a locally compact group. When $G$ is abelian, Cohen [1] characterized all of the idempotents in the measure algebra $\mathrm{M}(G)$. For nonabelian $G$, the idempotent probabilities were characterized by Kawada and Itô [3], while the contractive idempotents were characterized by Greenleaf [2]. We give an exact statement of their results in Theorem 0.1 below. For certain compact groups, the central idempotent measures were characterized by Rider [7], in a manner which is pleasingly reminiscent of Cohen's result on abelian groups. Rider points out a counterexample to his result when some assumptions are dropped. This has motivated our Example 1.3(i) below.

Discussion of contactive idempotents has been conducted in the setting of locally compact quantum groups by Neufang, Salmi, Skalski, and the present author [5].

Copyright 2016 by the Tusi Mathematical Research Group.

Received Mar. 15, 2015; Accepted Aug. 11, 2015.

2010 Mathematics Subject Classification. Primary 43A05; Secondary 43A77, 43A40.

Keywords. measure algebra, idempotent, groups of measures. 


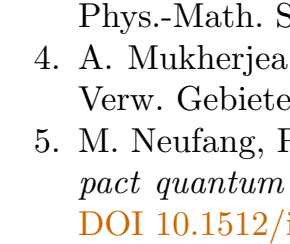

Ann. Funct. Anal. 7 (2016), no. 1, 136-149

http://dx.doi.org/10.1215/20088752-3428247

ISSN: 2008-8752 (electronic)

http://projecteuclid.org/afa

\title{
COMMUTING CONTRACTIVE IDEMPOTENTS IN MEASURE ALGEBRAS
}

\author{
NICO SPRONK \\ Dedicated to Professor Anthony To-Ming Lau, in honor of his contributions to, and leadership \\ in, the international abstract harmonic analysis community, the Canadian mathematical \\ community, and my career
}

Communicated by K. F. Taylor

\begin{abstract}
We determine when contractive idempotents in the measure algebra of a locally compact group commute. We consider a dynamical version of the same result. We also look at some properties of groups of measures whose identity is a contractive idempotent.
\end{abstract}

Let $G$ be a locally compact group. When $G$ is abelian, Cohen [1] characterized all of the idempotents in the measure algebra $\mathrm{M}(G)$. For nonabelian $G$, the idempotent probabilities were characterized by Kawada and Itô [3], while the contractive idempotents were characterized by Greenleaf [2]. We give an exact statement of their results in Theorem 0.1 below. For certain compact groups, the central idempotent measures were characterized by Rider [7], in a manner which is pleasingly reminiscent of Cohen's result on abelian groups. Rider points out a counterexample to his result when some assumptions are dropped. This has motivated our Example 1.3(i) below.

Discussion of contactive idempotents has been conducted in the setting of locally compact quantum groups by Neufang, Salmi, Skalski, and the present author [5].

Copyright 2016 by the Tusi Mathematical Research Group.

Received Mar. 15, 2015; Accepted Aug. 11, 2015.

2010 Mathematics Subject Classification. Primary 43A05; Secondary 43A77, 43A40.

Keywords. measure algebra, idempotent, groups of measures. 